\title{
1 Identification of snails and parasites of medical importance via convolutional neural network: an application for human schistosomiasis
}

\author{
Zac Yung-Chun Liu' ${ }^{1^{*}}$, Andy J. Chamberlin ${ }^{1^{*}}$, Pretom Shome ${ }^{1}$, Isabel J. Jones ${ }^{1}$, Gilles Riveau², \\ Raphael A. Ndione², Lydie Bandagny², Nicolas Jouanard ${ }^{2,3}$, Paul Van Eck ${ }^{4}$, Ton $\mathrm{Ngo}^{4}$, Susanne \\ H. Sokolow ${ }^{1,5}$, and Giulio A. De Leo ${ }^{1,6}$
}

Keywords: computer vision, Schistosomiasis, neglected tropical disease, deep learning, neural networks

Manuscript submission to PLOS Neglected Tropical Diseases

${ }^{1}$ Hopkins Marine Station, Stanford University, Pacific Grove, CA 93950, USA.

${ }^{2}$ Centre de Recherche Biomédicale Espoir pour la Santé, BP 226 Saint-Louis, Senegal.

3Station d'Innovation Aquacole (SIA), à Université Gaston Berger, Sanar, BP524 Saint-Louis, Senegal.

${ }^{4}$ IBM Silicon Valley Lab, San Jose, CA 95141, USA.

${ }^{5}$ Department of Ecology, Evolution, and Marine Biology and Marine Science Institute, University of California, Santa Barbara, CA 93106, USA.

${ }^{6}$ Woods Institute for the Environment, Stanford University, Pacific Grove, CA 93950, USA

*Send correspodence to: zacycliu@stanford.edu and achamb@stanford.edu

(1)




\section{Abstract}

Schistosomiasis is a debilitating parasitic disease infecting over 250 million people with nearly 800 million people at risk worldwide, primarily in sub-Saharan Africa. Transmission to humans involves freshwater snails as intermediate hosts, which are particularly prevalent in developing countries where dams and water resource projects have expanded freshwater snail habitat. At our study sites in the lower Senegal River Basin, we have collected more than 5,500 images of the 7 freshwater snail species (grouped into 4 categories) most frequently encountered in this aquatic ecosystem, 5 of which amplify and transmit either urinary or intestinal human schistosomiasis, with the other 2 species responsible for the transmission of less common parasitic diseases of humans and/or livestock. We have also collected over 5,100 images of 11 classes of trematodes, including human and non-human schistosomes. It takes a

44 great deal of training and expertise to accurately classify these organisms morphologically. In recent years, deep convolutional neural networks (CNNs) have proven to be highly efficient for

47 capacity for snail and parasite images and test our model's performance against 8 highly-trained

48 human parasitologists with experience taxonomically classifying snail and parasite species from

49 the Senegal River Basin in West Africa. We establish and train a single CNN end-to-end directly

50 from images with only pixels and labels as inputs. Applying this state-of-the-art algorithm, we

51 are able to classify images of 4 snail categories with $99.64 \%$ accuracy and images of 11

52 parasite categories with $88.14 \%$ accuracy, which rivals highly-trained human parasitologists.

53 The trained algorithm could next be deployed to mobile devices for use in remote field settings

54 by local technicians, and significantly improve monitoring snail and parasite occurrence in the

\section{5 field for disease control purposes.}

\section{Author Summary}


Schistosomiasis is a neglected tropical disease (NTD) infecting over 250 million people worldwide. The current approach to mitigate this disease in endemic regions is community or school-based mass drug administration. However, parasites are primarily transmitted through environmental reservoirs where freshwater snails serve as intermediate hosts. People use the contaminated water sources for their daily tasks and get re-infected after drug treatment.

63 Therefore, drug administration alone is not effective for schistosomiasis control in such high

64 transmission settings. Recent studies show that snail population control is essential to reduce

65 disease transmission risks. To discern between parasitic worms of humans in snails and those

66 of other non-human species is a necessary step to precisely quantify transmission risk for

67 human schistosomiasis. However, it takes a great deal of expertise to train lab and field

68 technicians to accurately classify snail and parasite species. Artificial intelligence (Al)-powered computer vision algorithms have recently proven to be highly efficient for image recognition tasks. We have collected thousands of snail and parasite images in Senegal during 2015-2018.

71 Using these images as training data, we developed an Al model that classifies images of 4 snail

72 categories and 11 parasite categories with, in some cases, higher accuracy than well-trained

73 human parasitologists. This model could next be deployed to mobile devices for use in remote

74 field settings to support local technicians to identify transmission hotspots and target control.

\section{Introduction}

Most human parasitic infections, such as schistosomiasis, onchocerciasis, lymphatic

78 filariasis, and malaria, are diseases of poverty: even while effective drugs to treat people exist,

79 the most affected communities are too poor to access them. Global health efforts have

80 attempted to fill this gap by delivering needed medication to rural communities in some of the

81 poorest countries through mass drug administration programs, a strategy that takes a practical

82 approach to control, treating whole communities, affordably, without regard to individual 
83 infection status. Yet because many parasites are transmitted through environmental reservoirs,

84 vectors, or intermediate hosts unaffected by this scheme, reinfection after treatment can be a

85 major impediment to achieving control using drugs alone. Thus, complementing human

86 treatment with transmission control, by identifying and eliminating hotspots of vectors and

87 reservoirs in the environment, could be most effective. Yet, this approach is severely hindered

88 by a lack of ecological research capacity in poor countries to identify, monitor, and affordably

89 eliminate relevant vectors and parasites with minimal environmental damage.

Human schistosomiasis is the second most important parasitic disease in the world

91 behind malaria, affecting over 250 million people in over 70 tropical and subtropical countries.

92 The majority of cases occur in sub-Saharan Africa, where, in recent decades, the expansion of

93 dams and water management infrastructure has resulted in the expansion of freshwater snail

94 habitat and human-water contact. These changes have benefited transmission of schistosoma

95 worms, which have a complex life cycle (Fig 1) involving: (1) a final vertebrate host (humans),

96 where the adult worms live and reproduce sexually, and (2) an intermediate freshwater snail

97 host where the larval stages of the worms develop via asexual reproduction. People are infected

98 through contact with water contaminated by free-living stages of the parasites that shed daily by

99 infected snails. Upon contact, the parasite penetrates skin and enters the bloodstream.

100 Therefore, infected snails in the freshwater environment are the source of rapid infection and re-

101 infection of humans in endemic areas [4]. And, despite the rise in preventive chemotherapy

102 campaigns in the last several decades, just as many people suffer from schistosomiasis today

103 as did 50 years ago [5].

104 Reducing snail populations using molluscicides has been an effective way to reduce

105 transmission of human schistosomiasis because snails serve as intermediate hosts of the

106 parasite [1, 2], but widespread mollusciciding has fallen out of favor because it is expensive and

107 can lead to non-target toxic effects in the environment [3]. New ways to treat environments 
safely (e.g. natural plant-based molluscicides, or biological control using natural enemies) and

to target treatments only where infected snails are abundant are desperately needed.

111 Figure 1. The life cycle of Schistosoma spp. parasites. The adult worms live and reproduce 112 sexually within the human host, releasing eggs into the environment that hatch and seek an 113 intermediate freshwater snail host, where the larval stages of the worms develop via asexual 114 reproduction. Cercariae are released from snails and seek human hosts, completing the 115 lifecycle.

In sub-Saharan Africa, there are two main species of schistosoma parasites that infect humans and use different snail species as an intermediate host: Biomphalaria (for Schistosoma mansoni) and Bulinus (for Schistosoma hematobium) [1]. Detecting the abundance and spatial distribution of both snail species and human schistosoma parasites is essential to design control

121 strategies aimed at reducing the environmental reservoir of the disease and/or administering

122 anthelminthic drugs where transmission risk is highest. Proper classification of snail species and

123 of parasites relevant to human health is the crucial first step to deploy effective control

124 strategies.

125 One of the most underrated tasks in the fight against schistosomiasis and other 126 helminthiases is accurate classification of snail and parasite species. Attempting this on the 127 basis of morphology is a labor-intensive, time-consuming job that requires a great deal of 128 training experience and expertise. Morphological differences between Bulinus spp. and 129 Biomphalaria spp. snails at the genus level are clear to any trained naturalist, but distinguishing 130 the specific snail species that are involved in the transmission of human schistosoma parasites 131 might be challenging for students and technicians in their early field and laboratory experience 132 or without continuing training. An additional challenge is that the same snail species that carry 133 human parasites can also be infected with a wide variety of livestock and wildlife parasites, 134 some of which are morphologically similar (and in some cases, even indistinguishable) to 
135 human schistosome larvae [6]. As a consequence, visual identification of parasite species

136 during snail dissection can be tricky even for experienced parasitologists. Misidentification of

137 snail infections as human schistosomes, when in fact they are cryptic species that do not harm

138 people, may interfere with cost-effectively deploying badly needed control in areas where

139 resources are limited to begin with.

140 To address this issue, we developed a computational method with the capacity to

141 automatically identify snails and parasites from photographs taken with a cell phone

142 camera. We apply convolutional neural networks (CNNs), a deep learning application in

143 computer vision, to identify the images and compare results to those of trained human

144 parasitologists.

Holmström et al. (2017) [7] conducted a proof-of-concept study on mobile digital

146 microscopy using deep learning for the detection of soil-transmitted helminthes and

147 Schistosoma haematobium eggs in human excreta, which could aid in medical diagnosis of

148 infected people, but cannot improve identification or mapping of high risk environments. Here,

149 we offer a new tool for automated detection and classification of larval parasites released from

150 medically relevant freshwater snails in Senegal. The goal of this study was to provide a proof of

151 concept that the application of the latest computer vision classification algorithms, powered by

152 artificial intelligence $(\mathrm{Al})$ technology, may help to improve snail and parasites identification in

153 capacity-limited, resource-poor areas where schistosomiasis is endemic.

155 Materials and Methods

156 Datasets

157 During environmental monitoring of snails and parasites in the Senegal River Basin

158 during 2016-2018, we collected 5,543 images of four genera of medically important and

159 common freshwater snails -- (1) Bulinus, (2) Biomphalaria, (3) Lymnaea, and (4) Melanoides -- 
and 5,140 images of parasitic cercariae, from 11 morphospecies liberated from the Biomphalaria and Bulinus spp. snails encountered and dissected. Table 1 summarizes the

162 numbers of images in each category and Fig 2 shows examples for each snail and parasite

163 category in our dataset. Snail photographs were taken by mobile phone devices and cameras

164 with similar resolution (at least $1024 \times 768$ pixels) and taken from similar angles, zoom,

165 backgrounds, and lighting; while parasite images were taken from the same cell phones through

166 dissecting microscopes, with the same uniformity. In addition, all parasite images were

167 processed to grayscale. The removal of color was performed to further decrease lighting and

168 time of day artifacts that could affect computer vision results. The detailed protocols for image

169 collection and quality control are provided in the supplemental materials (see S1 Appendix).

170 Initial identification of snails and parasites was performed by trained parasitologists in the field.

171 Cases of disagreement between specialists were resolved.

Accuracy of field identifications were verified by molecular barcoding technique: at the

173 time of shedding or dissection, in all fork-tailed cercariae liberated from snails were placed

174 individually on WhatmanFTA ${ }^{\odot}$ cards and sequenced to distinguish human-infective Schistosoma

175 haematobium and S. haematobium-bovis hybrids from S. bovis (schistosome parasite of cattle)

176 and other non-human furcocercous (forktailed) trematode species [8]. The identification of the

177 parasites was based on multi-locus analyses with one mitochondrial (cox1) and two nuclear

178 (ITS1+2 and 18S) genes [9]. Cercariae on FTA cards were accessioned into the

179 Schistosomiasis Collection at the Natural History Museum (SCAN) [10].

181 Table 1. Summary of numbers of images for each snail and parasite species; numbers of 182 images for the split of training (80\%) and validation set $(20 \%)$.

\begin{tabular}{lcccc}
\hline Snail class & Biomphalaria & Bulinus & Lymnaea & Melanoides \\
\hline Number of images & 466 & 4215 & 725 & 137 \\
\hline
\end{tabular}




\begin{tabular}{lcccc}
\hline Training & 374 & 3372 & 580 & 109 \\
Validation & 92 & 843 & 145 & 28 \\
\hline
\end{tabular}

\begin{tabular}{lccccccccccc} 
Parasite class & HS & NHS1 & NHS2 & AM & BO & EC & GY & ME & PP & PT & XI \\
\hline Number of images & 1008 & 638 & 107 & 442 & 224 & 332 & 152 & 196 & 806 & 231 & 1004 \\
Training & 806 & 510 & 86 & 354 & 179 & 266 & 121 & 156 & 645 & 185 & 803 \\
Validation & 202 & 128 & 21 & 88 & 45 & 66 & 31 & 40 & 161 & 46 & 201 \\
& & & & & & & & & & &
\end{tabular}

Abbreviations of parasite classes as follow, HS: Human-schisto, NHS1: Nonhuman- schisto forktail type I, NHS2: Nonhuman- schisto forktail type II, AM: Amphistome, BO: Bovis, EC: Echino, GY: Gymno, ME: Metacerc, PP: Parapleurolophocercous, PT: Parthenitae, XIXiphidiocercariae.

Figure 2. Image examples for snail and parasite classes. For snail classes, A-1 and A-2: Biomphalaria. B-1 and B-2: Bulinus. C-1 and C-2: Lymnaea. D-1 and D-2: Melanoides. For parasite classes, HS: Human-schisto, NHS1: Nonhuman- schisto forktail type I, NHS2: Nonhuman- schisto forktail type II, AM: Amphistome, BO: Bovis, EC: Echino, GY: Gymno, ME: Metacerc, PP: Parapleurolophocercous, PT: Parthenitae, XI-Xiphidiocercariae.

All Biomphalaria and Bulinus spp. snails were also identified to morphospecies by both

197 trained parasitologists and genetic barcoding. Total genomic DNA was isolated from a small

198 amount of snail tissue using the DNeasy Blood and Tissue kit (Qiagen, UK) according to

199 manufacturer's instructions. Amplification of a partial cytochrome oxidase 1 (cox 1) sequence

200 was carried out on snail vouchers [11]. PCR and sequencing conditions were chosen as

201 previously published [12]. Sequencing was performed on an Applied Biosystems 3730XL

202 analyser (Life Technologies, UK) [9]. The field ID guidelines for morphologies of snails and

203 parasites are provided in the supplemental materials (see S2 Appendix). 
For imagery of snails and parasites, we strategically avoided extensive processing of images, since the ultimate goal is to create a CNN capable of classification of images of variable quality and resolution. We anticipate that many technicians will be taking pictures directly from

207 mobile devices in the field. So, in the preparation of our training data, images that were severely

208 blurry were removed from the test and validation sets, but were still used in training. For each

209 class of snails and parasites, we randomly divided the dataset into training and validation set,

210 containing $80 \%$ and $20 \%$ of the total images, respectively (Table 1 ). There was no overlap

211 between the training set and the validation set.

\section{Training Algorithm}

213 Deep learning algorithms with recent advances in computation and large datasets have

214 been shown to be comparable with, and even exceed, human performance in various object

215 recognition and computer vision tasks, including applications to diagnose human disease (e.g.,

216 ImageNet challenge [13], breast cancer histology images [14], and skin cancer images [15]).

217 Convolutional neural networks (CNNs) learn key features directly from the training images by

218 the optimization of the classification loss function $[16,17]$ and therefore have minimal need for a

219 priori knowledge to design a classification system. Thus, the performance is less biased by the

220 assumptions of the researchers $[13,18]$.

221 In this study, we utilized VGG16 pre-trained model [19], a 16-layer CNN architecture,

222 which was trained on approximately 1.28 million images with 1,000 categories. VGG16 was

223 used by the VGG team (Visual Geometry group at the University of Oxford) in the 2014

224 ImageNet Large Scale Visual Recognition Challenge. We trained VGG16 on our snail and

225 parasite images using transfer learning [20]. Transfer learning is defined as exporting

226 knowledge from previously learned sources to a target task [21]. The network architecture

227 established here has convolutional-pooling layer pairs (max-pooling), followed by a fully228 connected network [17]. The network architecture is illustrated in S1 Fig. The CNN is trained 
end-to-end directly from image labels and raw pixels, with a VGG16 network for photographic

230 images of snails and another separated network for microscopic images of parasites.

We adopted the training patches as $64 \times 64$ pixels for input layers in the consistent with

232 VGG16 network architecture, to ensure the patch size was sufficient to cover the relevant

233 structures and morphologies of snail and parasites. We first initialized the weights with the

234 VGG-16 pre-trained on ImageNet dataset, then froze the bottom of the network and just trained

235 the top of the VGG-16 convolutional networks (S1 Fig). The fully-connected layers were

236 composed of Rectified Linear Units (i.e., the ReLU activation function), to avoid the vanishing

237 gradients and to improve the training speed [22]. The output layer was composed of 4 neurons

238 for snail classification, corresponding to each of the four classes that are normalized with a

239 softmax activation function. For the parasite classification task, 11 neurons were set up in the

240 same manner. The model was trained with $80 \%$ of the training set, and validated on the

241 remaining images not used for training. Note that the validation set is randomly selected for

242 each epoch, which is the measure of the number of times all of the training images are used

243 once to update the network weights [17]. The network weights were initialized randomly, and an

244 adaptive learning rate gradient-descent back-propagation algorithm [22] was used for weight

245 update. Here we selected categorical cross-entropy as a loss function in the model. In these two

246 classification tasks, the CNN outputs a probability distribution over 4 classes of snails and 11

247 classes of parasites.

248 Since our dataset was rather small comparing with recent studies using CNNs for image

249 recognition tasks [13], here we applied the techniques of dropout and regularization to

250 overcome the overfitting of training data [17]. We implemented the CNN model with VGG16

251 network in a Python environment with the Keras package [23], and Google's deep learning

252 framework, TensorFlow [24].

\section{Data Augmentation}


need to be identified are equally represented in the dataset [17]. Our dataset was unbalanced:

for example, Bulinus snails comprised about $75 \%$ of total snail images, since this snail genus

257 was most common in the field sites. Thus, we applied a data augmentation approach and used

258 rotation and shifting [25] to generate more images for the snail and parasites species that were

259 underrepresented in the dataset. Mirroring was not used Bulinus and Lymnaea spp due to the

260 diagnostic value of their coil orientation (i.e. Bulinus is sinistral and Lymnaea is dextral). We also

261 applied Gaussian noise to the background of parasite training images [26] to ensure that the

262 model did not learn background artifacts, but focused only on learning the morphologies of the

263 parasite objects. In this study, we used a Keras defined generator for automating data

264 augmentation [23]; every item of every batch was randomly changed according to the following

265 settings: (1) rotation range $=20,(2)$ width shift range $=0.2,(3)$ height shift range $=0.2$, and (4)

266 shear range $=0.15$. In practice, rotations and shifting allowed us to increase the size of the

267 dataset without deteriorating its quality. Data augmentation used here further improved the

268 datasets and CNN predicting performance.

269 Model deployment

271 network weights to establish a web application for inference using TensorFlow.js [27], which is a

272 library for executing machine learning algorithms in JavaScript. TensorFlow.js is compatible with

273 the Python-based TensorFlow and Keras APIs, allowing our Keras model to be converted to a

274 JavaScript format that can be run in a web browser. This makes it accessible on any device with

275 a modern browser including both smartphones and common laptop computers. Using browser

276 storage and caching APIs, the web application can even be used in areas with limited or no

277 internet connectivity.

278 Ethics Statement 
280 Espoir pour la Santé in Senegal who obtained the permission to conduct the field snail

281 collection, from The Direction de l'Environement et des Etablissements Classés with the

282 identification number "N002302 MEDD/DEEC/yn". In this study, we use photos of snails for the

283 machine learning model training and validation, therefore, there was no direct use of snails in

284 the field environment.

\section{Results}

We evaluated our CNN model's performance with metrics of accuracy, sensitivity, specificity, and F1 score on the test set. With the optimized CNN architecture, we obtained 99.6\% accuracy (proportion of correct classifications, either true positive or true negative) for the 4 snail genera and $88.14 \%$ for the 11 parasite morphospecies. The optimized dropout rates for 291 the convolutional layer [17] for the snail dataset was 0.6 and for parasite dataset was 0.75 . For

292 the parasite set, we ran a second analysis with only 3 classes of parasites relevant to map risk 293 for schistosomiasis transmission, namely: human schistosomes, non-human forktail cercariae, 294 and other trematode morphotypes. The overall accuracy for the 3 parasite classes increased to $92.41 \%$. The sensitivity of distinguishing human schistosomes from non-human parasites was parasite morphotypes, along with details of true positive (TP), true negative (TN), false positive

298 (FP), and false negative (FN) outcomes.

Figure 3: Confusion matrix of classification on test set. (a) results of snail image set, labels:

301 0-Biomphalaria, 1: Bulinus, 2: Lymnaea, 3: Melanoides. (b) results of parasite image set, labels:

302 0: Amphistome, 1: Bovis, 2: Echino, 3: Gymno, 4: Human-schisto, 5: Metacerc, 6:

303 Parapleurolophocercous, 7: Parthenitae, 8: Nonhuman- schisto forktail type I, 9: Nonhuman304 schisto forktail type II, 10: Xiphidiocercariae. (c) Combining other trematodes as one category, 305 labels: 0: Schisto, 1: Non-human forktail type I, type II, and Bovis, 2: Other trematodes. 

calculated as follows:

311 precision $=\mathrm{TP} /(\mathrm{TP}+\mathrm{FP})$

$\mathrm{F} 1$ score $=\left(\frac{\text { recall }^{-1}+\text { precision }^{-1}}{2}\right)^{-1}$

313 Sensitivity measures the proportion of positives that are correctly identified; in our case,

314 we focused on the percentage of human schistosomes correctly identified. Specificity measures

315 the proportion of negatives that are correctly identified; in our case, we focused on the

316 percentage of non-human forktailed cercariae (some of which can potentially be visually similar

317 and hard to distinguish from human schistosomes) that are correctly identified as non-human

318 schistosomes. Precision is a measure of a classifier's exactness, while recall is a measure of a

319 classifier's completeness. Low precision indicates a large number of false positives, while low

320 recall indicates many false negatives. F1 score conveys the balance between precision and

321 recall and is defined as the harmonic mean of precision and sensitivity. Our results showed that

322 the CNN produced high sensitivity and specificity (> 84\%) as well as F1 score $(>0.84)$ in all

323 categories. The metrics to measure classification performances are shown in Table 2 and

324 demonstrate the robustness of the CNN training algorithm for the tasks of image recognition for 325 both snails and parasites, despite the low sample size we had to work with.

327 Table 2. Results of sensitivity(\%) and specificity(\%) of CNN model.TP: true positive, TN: true 328 negative, FP: false positive, FN: false negative. 


\begin{tabular}{lllllll}
\hline Snail class & TP & TN & FP & FN & sensitivity(\%) & specificity(\%)
\end{tabular}

\begin{tabular}{lrrrrrrr}
\hline Biomphalaria & 92 & 1020 & 0 & 0 & 100.00 & 100.00 & 1.00 \\
Bulinus & 843 & 265 & 4 & 0 & 100.00 & 98.51 & 0.99 \\
Lymnaea & 145 & 963 & 0 & 4 & 97.31 & 100.00 & 0.99 \\
Melanoides & 28 & 1084 & 0 & 0 & 100.00 & 100.00 & 1.00 \\
\hline
\end{tabular}

\begin{tabular}{llllllll}
\hline $\begin{array}{l}\text { Parasite } \\
\text { class }\end{array}$ & TP & TN & FP & FN & sensitivity(\%) & specificity(\%) & F1 socre
\end{tabular}

\begin{tabular}{lccccccc}
\hline Schisto & 169 & 795 & 32 & 33 & 83.66 & 96.13 & 0.84 \\
$\begin{array}{l}\text { Non-human } \\
\text { forktail }\end{array}$ & 164 & 820 & 15 & 30 & 84.53 & 99.51 & 0.91 \\
$\begin{array}{l}\text { Other } \\
\text { trematodes }\end{array}$ & 618 & 365 & 31 & 15 & 97.63 & 92.17 & 0.96 \\
\hline
\end{tabular}

\section{Comparison with human parasitologist performance}

332 To validate our deep learning approach, we compared the direct performance of the

$333 \mathrm{CNN}$ to 8 human parasitology experts. For each image, the parasitologists were asked to

334 identify the category of the snails and parasites. We prepared 30 snail images from among the 4

335 categories and 120 parasite images from among the 11 morphotypes in the computer's

336 validation sets. For each test, previously unseen, molecularly verified images of trematode

337 cercariae and snails were displayed, and parasitologists are asked to identify them from among

338 the same categories of snails and parasites that the computer vision algorithm trained on.

339 Identification guidelines were provided along with the quiz. The sample quiz is included in 
340 supplement materials (see S3 Appendix). The metrics to measure human parasitologists'

341 performances and compare with the computer's, such as sensitivity, specificity, rand F1 score

342 are shown in Table 3 and Fig 4. The CNN generated a malignancy probability $P$ per image. We

343 then fix a threshold probability $t$ such that the prediction $\hat{y}$ for any image is $\hat{y}=P \geq t$, and the

344 blue curve (Fig 4) is drawn by sweeping t in the interval $0-1$. The area under the curve (AUC) is

345 the CNN's measure of performance, with a maximum value of 1 . The AUC for human

346 schistosomes, non-human forktailed cercariae, and other trematodes were 0.96, 0.95, 0.98,

347 respectively. The CNN achieved superior performance to a parasitologist if the sensitivity-

348 specificity point of the parasitologist lies below the blue curve, which most do. Note that

349 sensitivity and specificity of human parasitologists to correctly distinguish cryptic human

350 schistosome and non-human-schistosome species (Schistosoma bovis) was significantly lower

351 than the CNN.

353 Table 3. Results of sensitivity(\%) and specificity(\%) of 8 human parasitologists

\begin{tabular}{lccccccc}
\hline Snail class & TP & TN & FP & FN & sensitivity(\%) & specificity(\%) & F1 score \\
\hline Biomphalaria & 2 & 8 & 0 & 0 & 100.00 & 100.00 & 1.00 \\
Bulinus & 3 & 7 & 0 & 0 & 100.00 & 100.00 & 1.00 \\
Lymnaea & 3 & 7 & 0 & 0 & 100.00 & 100.00 & 1.00 \\
Melanoides & 2 & 8 & 0 & 0 & 100.00 & 100.00 & 1.00 \\
\hline
\end{tabular}

354

\begin{tabular}{|c|c|c|c|c|c|c|c|}
\hline $\begin{array}{l}\text { Parasite } \\
\text { class }\end{array}$ & TP & $\mathrm{TN}$ & FP & $\mathrm{FN}$ & sensitivity(\%) & specificity(\%) & F1 socre \\
\hline Schisto & 15 & 99 & 5 & 1 & 93.75 & 95.19 & 0.83 \\
\hline $\begin{array}{l}\text { Non-human } \\
\text { forktail }\end{array}$ & 19 & 95 & 3 & 5 & 79.17 & 96.94 & 0.83 \\
\hline
\end{tabular}




\begin{tabular}{llllllll}
\hline $\begin{array}{l}\text { Other } \\
\text { trematodes }\end{array}$ & 78 & 40 & 0 & 2 & 97.50 & 100.00 & 0.98
\end{tabular}

356 Figure 4: Comparison of classification performance with CNN and parasitologist. The

357 CNN outperforms any parasitologist whose sensitivity and specificity point (in red color) falls

358 below the blue curve of the CNN. The green points represent the average of the parasitologists

359 (average sensitivity and specificity of all red points), with error bars denoting one standard

360 deviation. We simplify the 11 classes of parasite to only 3 classes of interest for schistosomiasis

361 environmental risk mapping: (a) human schistosomes, (b) non-human forktail cercariae, and (c)

362 other trematode morphotypes. The area under the curve (AUC) for each case is over 95\%. The

363 CNN achieves superior performance to a parasitologist if the sensitivity-specificity point of the

364 parasitologist lies below the blue curve.

\section{Discussion}

In this work we developed and presented a deep learning model that is capable of accurately classifying images of human Schistosomiasis larval parasites and intermediate host snails to benefit environmental monitoring and control of this debilitating disease. The accuracy of our models matched and in most cases exceeded highly trained field experts that we tested it

371 against. Our convolutional neural network (CNN) classification performed better for snail

372 recognition than for parasite recognition. This is because snail morphologies are generally more

373 distinct, and it is easier to take high quality pictures of snails than parasites. This decreases the

374 risk of erroneous classification of snails, as reflected by the small number of false positives and

375 false negatives in our model evaluation. Parasites, on the other hand, show more complex

376 morphologies and variation, even within a species, which makes discrimination of trematode

377 cercariae more challenging, as reflected by the slightly large number of false negative and false 378 positive classification.

379 A benefit of CNN is that models can be trained with rather limited image datasets using 380 data augmentation approaches. Because we detected a low number of morphologically similar 
381 forked-tail human and non-human schistosoma parasites, we used rotation and shifting

382 methods to effectively increase the number of images used in training. We had the advantage of

383 also having video footage of living, mobile cercariae. As in our case, in the future, collecting

384 video imagery of parasites to generate many unique still-images of cercariaes an easy way to

385 increase samples for training CNN models.

In addition to building a deep learning model, we have also developed a workflow for

387 image collection and quality control for other researchers to follow, to quickly build an image

388 database that can facilitate identification of medically-important snails and their parasites with

389 the click of a cell phone and a pre-trained computer vision model. The protocol for image

390 collection is provided in the supplemental materials (see Appendix S2). We hope that user

391 photos can ultimately be used to create a global database of medically important snail and

392 parasite species, and the species they co-exist with that are not relevant to human health,

393 useful for further model training. When collecting the samples in the field, we also suggest that

394 GPS locations be recorded, which can facilitate the application of these data for precision

395 mapping of snail and parasite distribution in space and time. We take inspiration from online

396 platforms that encourage citizen science and crowdsourcing activities, similar to iNaturalist,

397 NaturNet, MycoMap, etc. iNaturalist, for example, has the image classification capacity to

398 immediately suggest an object name right after a user has uploaded an image to the platform.

399 To encourage the development of our specific platform and for translating it for use on

400 other medically-relevant parasites, vectors, and non-human hosts found in the environment, our

401 code, image sets, and neural network weights are provided in a public repository, which also

402 includes detailed documentation to assist researchers in following our workflow to (1) reproduce

403 the results, and (2) build new models using their own image datasets. We have also deployed

404 our classification model to a web application that allows researchers to select their own images

405 of snail and parasites to obtain the classification prediction, which is the probability of an object

406 of belonging to a specific class. Even though it takes a considerable computing power to 
properly train a CNN image classification model, once trained and deployed, a CNN can provide a result in a fraction of a second on a smartphone and a common laptop computer. recognition tasks for classification of medically relevant snails and their parasitic infections. We

411 apply a computer vision model, using a single convolutional neural network trained on a

412 relatively low number of cell-phone and dissecting microscope acquired images. Thus, we have

413 developed a proof of concept for this technology to be applied in resource-poor settings where

414 schistosomiasis is endemic and where identification of hotspots of transmission is desperately

415 needed to target interventions. Our model matched the performance of 8 highly trained human

416 parasitologists familiar with snail and parasite diversity in the study region. In light of its success,

417 we've deployed our product as a publically-accessible web application. In the future this method

418 could be deployed on mobile devices with minimal cost, and holds the potential for substantial

419 improvement for monitoring and identifying snail and schistosomiasis hotspots. Deep learning is

420 a powerful tool that can help fill the capacity gap limiting our understanding of the environmental

421 components of transmission for more affordable, efficient, and effective control of neglected

422 tropical diseases.

\section{Acknowledgement}

This work was supported by a grant from the Bill and Melinda Gates Foundation

426 (OPP1114050), an Environmental Ventures Program grant from the Stanford University Woods

427 Institute for the Environment, a SEED grant from the Freeman Spogli Institute at Stanford

428 University, a Human Centered Artificial Intelligence grant from Stanford University, a grant from

429 the National Institutes of Health (R01TW010286), and a grant from the National Science

430 Foundation (1414102). We thank Tim White and Richard Grewelle for their suggestions and

431 comments for this project. We thank the parasitologists who participated in the quiz that was 
432 designed to compare the performances of $\mathrm{CNN}$ and human experts on snails and parasites

433 image classification. We also thank IBM open source group, especially Yi Hong Wang, Chin

434 Huang, Ted Chang, Catherine Diep, Winnie Tsang, and Thomas Truong for providing guidance,

435 education, and cloud resources to establish the CNN model deployment process and host the

436 web application. Open Source code packages, image dataset, and web application can be

437 accessed via this link (https://github.com/deleo-lab/schisto-parasite-classification).

\section{Author Contributions}

440 ZYCL and AC built, trained, and validated the CNN model.

441 AC, IJJ, and SHS organized and labeled the image dataset.

442 AC, IJJ, SHS, GR, NJ, RN, and GADL conducted the field works.

443 PS performed the initial training and testing.

444 PVE and TN deployed the CNN model and created the web application.

445 GADL conceived the idea and supervised the project in all perspectives.

\section{References}

448 1. Sokolow SH, Huttinger E, Jouanard N, Hsieh MH, Lafferty KD, Kuris AM, Riveau G, Senghor S, Thiam C, N'Diaye A, Faye DS. Reduced transmission of human schistosomiasis after restoration of a native river prawn that preys on the snail intermediate host. Proceedings of the National Academy of Sciences. 2015 Aug 4;112(31):9650-5.

2. Sokolow SH, Wood CL, Jones IJ, Swartz SJ, Lopez M, Hsieh MH, Lafferty KD, Kuris AM, Rickards C, De Leo GA. Global assessment of schistosomiasis control over the past century shows targeting the snail intermediate host works best. PLoS neglected tropical diseases. 2016 Jul 21;10(7):e0004794.

3. Sokolow SH, Jones IJ, Jocque M, La D, Cords O, Knight A, Lund A, Wood CL, Lafferty KD, Hoover CM, Collender PA. Nearly 400 million people are at higher risk of schistosomiasis because dams block the migration of snail-eating river prawns. 

24;372(1722):20160127.

4. Steinmann P, Keiser J, Bos R, Tanner M, Utzinger J. Schistosomiasis and water resources development: systematic review, meta-analysis, and estimates of people at risk. The Lancet infectious diseases. 2006 Jul 1;6(7):411-25.

5. Sokolow SH, Wood CL, Jones IJ, Swartz SJ, Lopez M, Hsieh MH, Lafferty KD, Kuris AM, Rickards C, De Leo GA. Global assessment of schistosomiasis control over the past century shows targeting the snail intermediate host works best. PLoS neglected tropical diseases. 2016 Jul 21;10(7):e0004794.

6. Abbasi I, King CH, Sturrock RF, Kariuki C, Muchiri E, Hamburger J. Differentiation of Schistosoma haematobium from related schistosomes by PCR amplifying an interrepeat sequence. The American journal of tropical medicine and hygiene. 2007 May 1;76(5):950-5.

7. Holmström $\mathrm{O}$, Linder $\mathrm{N}$, Ngasala $\mathrm{B}$, Mårtensson $\mathrm{A}$, Linder $\mathrm{E}$, Lundin $\mathrm{M}$, Moilanen $\mathrm{H}$, Suutala A, Diwan V, Lundin J. Point-of-care mobile digital microscopy and deep learning for the detection of soil-transmitted helminths and Schistosoma haematobium. Global health action. 2017 Jun 30;10(sup3):1337325.

8. Webster BL, Rabone M, Pennance T, Emery AM, Allan F, Gouvras A, Knopp S, Garba A, Hamidou AA, Mohammed KA, Ame SM. Development of novel multiplex microsatellite polymerase chain reactions to enable high-throughput population genetic studies of Schistosoma haematobium. Parasites \& vectors. 2015 Dec;8(1):432.

9. Wood CL, Sokolow SH, Jones IJ, Chamberlin AJ, Lafferty KD, Juris AM, Jocques M, Hopkins S, Adams G, Schneider M, Buck JC, Lund A, Garcia-Vedrenne AE, Fiorenza E, Rohr JR, Allan F, Webster B, Rabone M, Webster JP, Bandagny L, Ndione R, Senghor S, Schacht A-M, Jouanard N, Riveau G, and De Leo GA. Precision mapping of snail habitat provides a powerful indicator of human schistosomiasis transmission. In reviewProceedings of the National Academy of Sciences. 2019 June. (SCAN). Parasites \& vectors. 2012 Dec;5(1):185.

11. Vrijenhoek R. DNA primers for amplification of mitochondrial cytochrome c oxidase subunit I from diverse metazoan invertebrates. Mol Mar Biol Biotechnol. 1994;3(5):2949.

12. Kane RA, Stothard JR, Emery AM, Rollinson D. Molecular characterization of freshwater snails in the genus Bulinus: a role for barcodes?. Parasites \& Vectors. 2008 Dec;1(1):15. 
13. Russakovsky O, Deng J, Su H, Krause J, Satheesh S, Ma S, Huang Z, Karpathy A, Khosla A, Bernstein M, Berg AC. Imagenet large scale visual recognition challenge. International journal of computer vision. 2015 Dec 1;115(3):211-52.

14. Araújo T, Aresta G, Castro E, Rouco J, Aguiar P, Eloy C, Polónia A, Campilho A. Classification of breast cancer histology images using convolutional neural networks. PloS one. 2017 Jun 1;12(6):e0177544.

15. Esteva A, Kuprel B, Novoa RA, Ko J, Swetter SM, Blau HM, Thrun S. Dermatologistlevel classification of skin cancer with deep neural networks. Nature. 2017 Feb;542(7639):115.

16. LeCun Y, Bengio Y. Convolutional networks for images, speech, and time series. The handbook of brain theory and neural networks. 1995 Apr;3361(10):1995.

18. Spanhol FA, Oliveira LS, Petitjean C, Heutte L. Breast cancer histopathological image classification using convolutional neural networks. In2016 international joint conference on neural networks (IJCNN) 2016 Jul 24 (pp. 2560-2567). IEEE.

19. Simonyan K, Zisserman A. Very deep convolutional networks for large-scale image recognition. arXiv preprint arXiv:1409.1556. 2014 Sep 4.

20. Pan SJ, Yang Q. A survey on transfer learning. IEEE Transactions on knowledge and data engineering. 2010 Oct;22(10):1345-59.

21. Bahadori MT, Liu Y, Zhang D. A general framework for scalable transductive transfer learning. Knowledge and information systems. 2014 Jan 1;38(1):61-83.

22. Nair V, Hinton GE. Rectified linear units improve restricted boltzmann machines. InProceedings of the 27th international conference on machine learning (ICML-10) 2010 (pp. 807-814).

23. Chollet, F. and others (2015). Keras. https://github.com/keras-team/keras

24. Abadi M, Barham P, Chen J, Chen Z, Davis A, Dean J, Devin M, Ghemawat S, Irving G, Isard M, Kudlur M. Tensorflow: A system for large-scale machine learning. In12th \{USENIX\} Symposium on Operating Systems Design and Implementation (\{OSDI\} 16) 2016 (pp. 265-283). single images. arXiv preprint arXiv:1312.5242. 2013 Dec 18. binary weights during propagations. InAdvances in neural information processing systems 2015 (pp. 3123-3131). 
27. Smilkov D, Thorat N, Assogba Y, Yuan A, Kreeger N, Yu P, Zhang K, Cai S, Nielsen E, Soergel D, Bileschi S. TensorFlow. js: Machine Learning for the Web and Beyond. arXiv preprint arXiv:1901.05350. 2019 Jan 16.

\section{Supporting Information Legends}

\section{Supplement Figure S1}

534 VGG-16 architecture illustration.

535

536 Supplement Appendix

537 S1. Protocols for image collection and quality control

538 S2. Field ID guidelines for morphologies of snails and parasites

539 S3. Sample quiz on snail and parasite classification for human parasitologists 


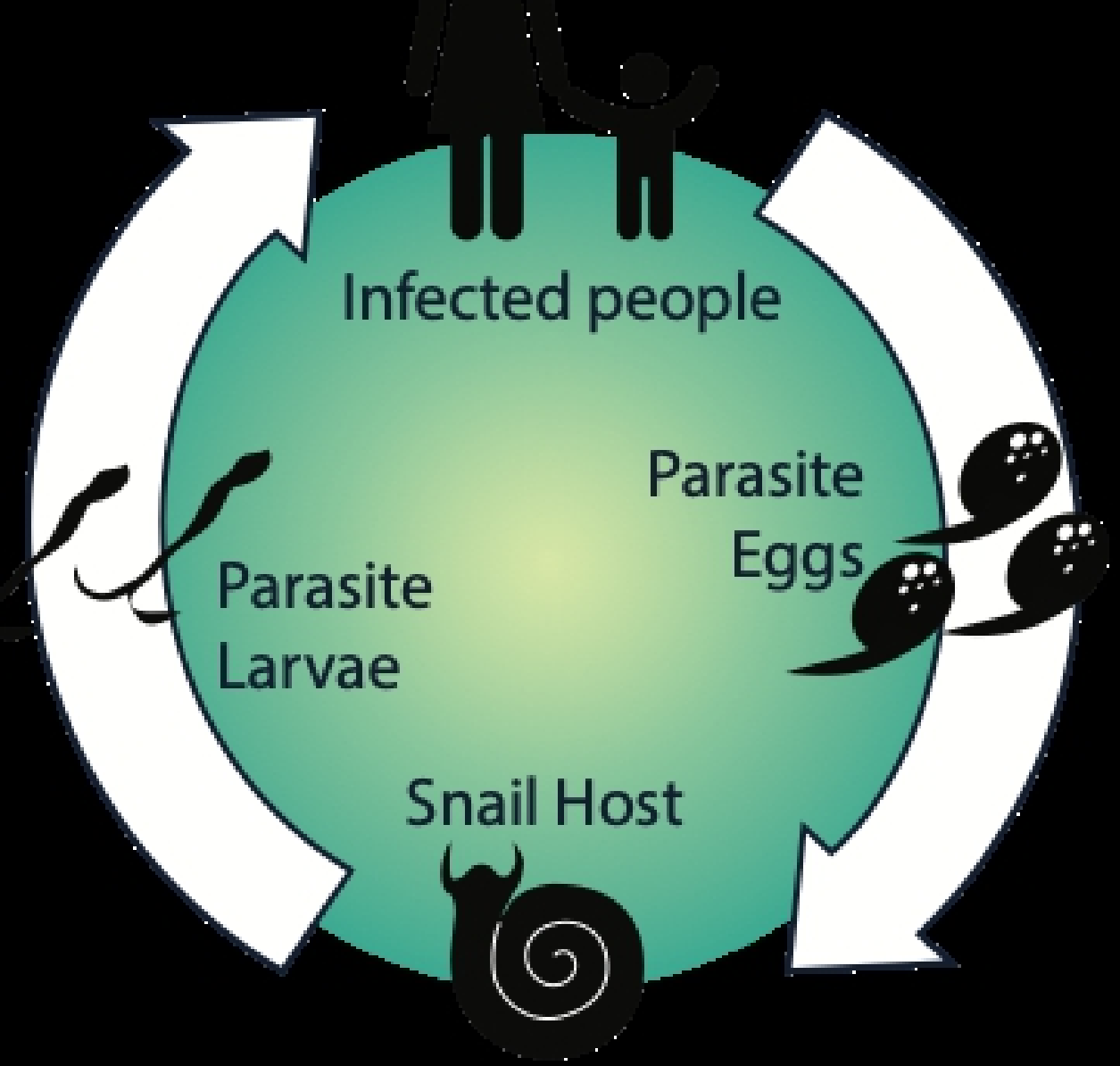

Figure 1 
(a) Snail images Test set

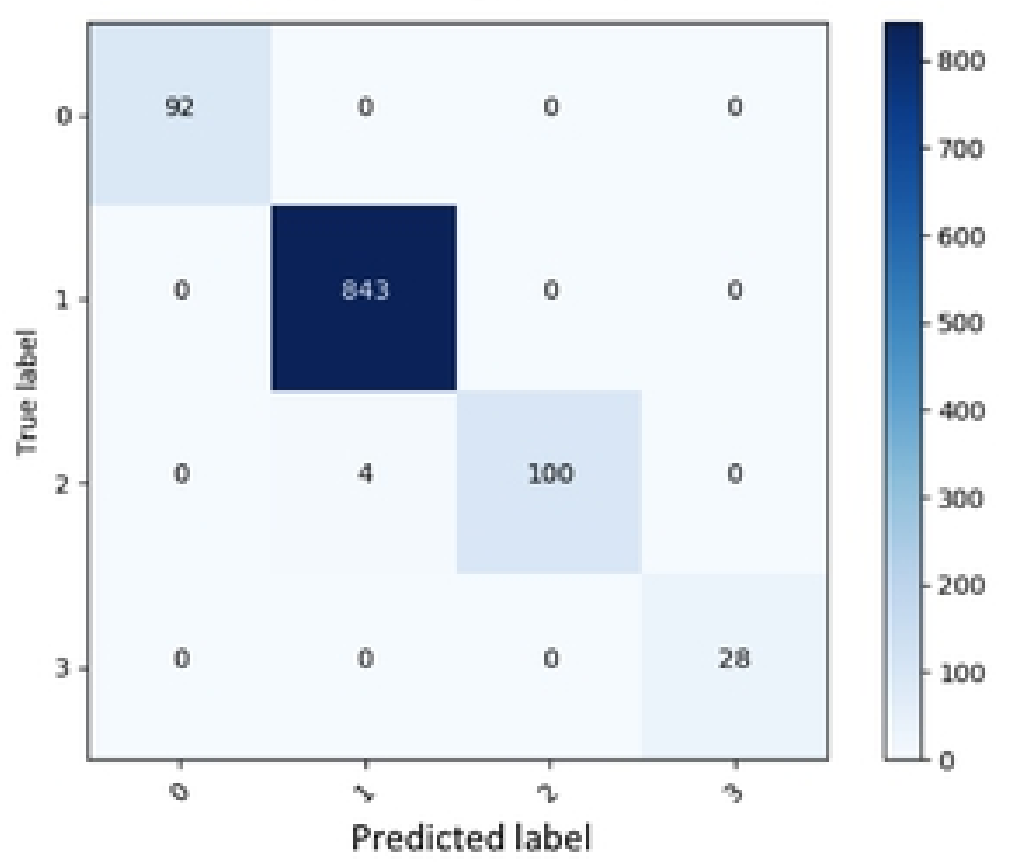

\section{(b) Parasite images Test set}

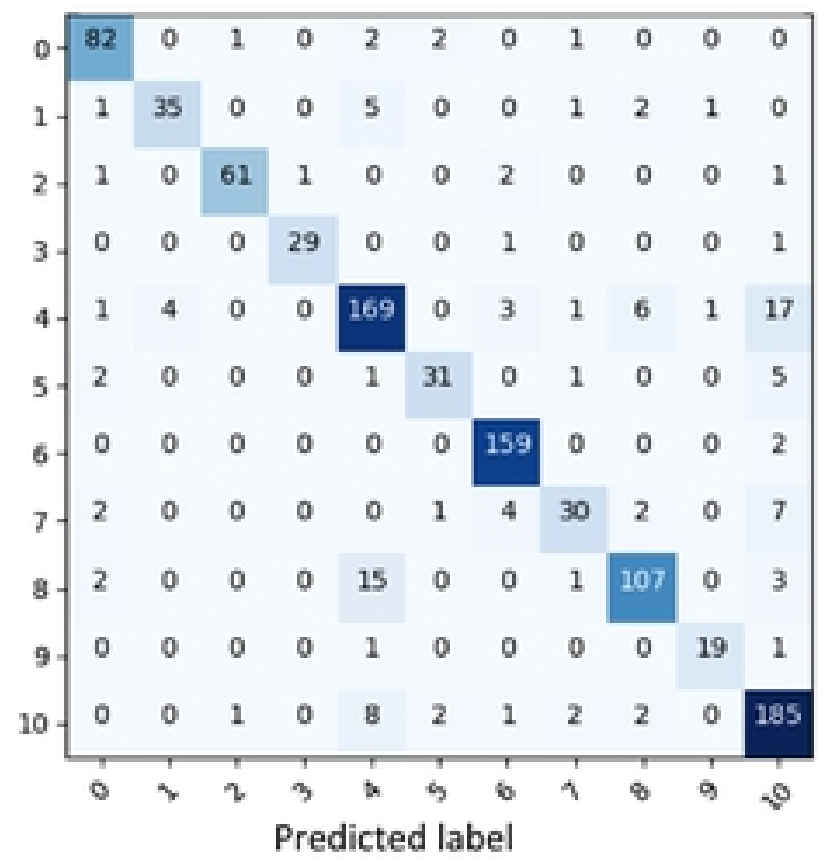

(c) Parasites images Test set- Schisto/ Non-Schisto
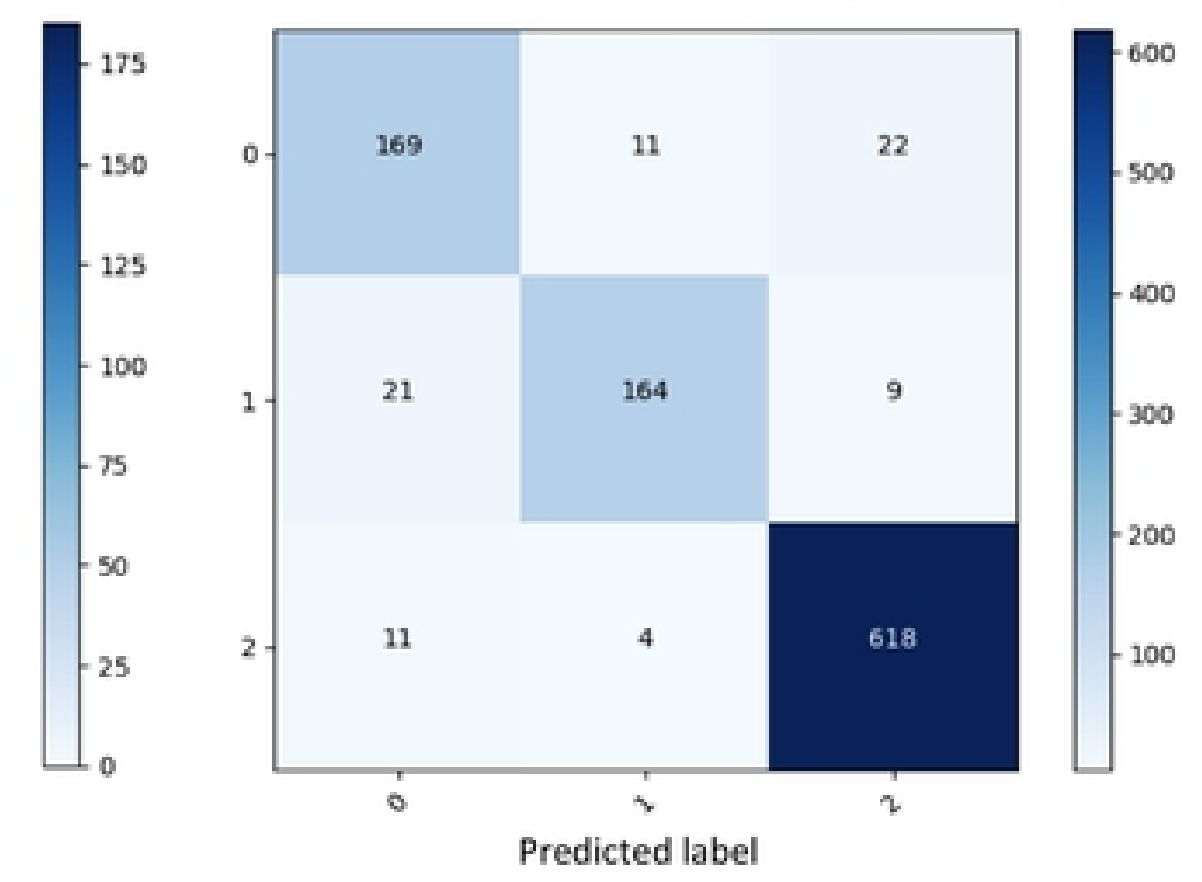

Figure 3 
Human Schisto

1029 images (CNN); 120 images (Parasitologists)

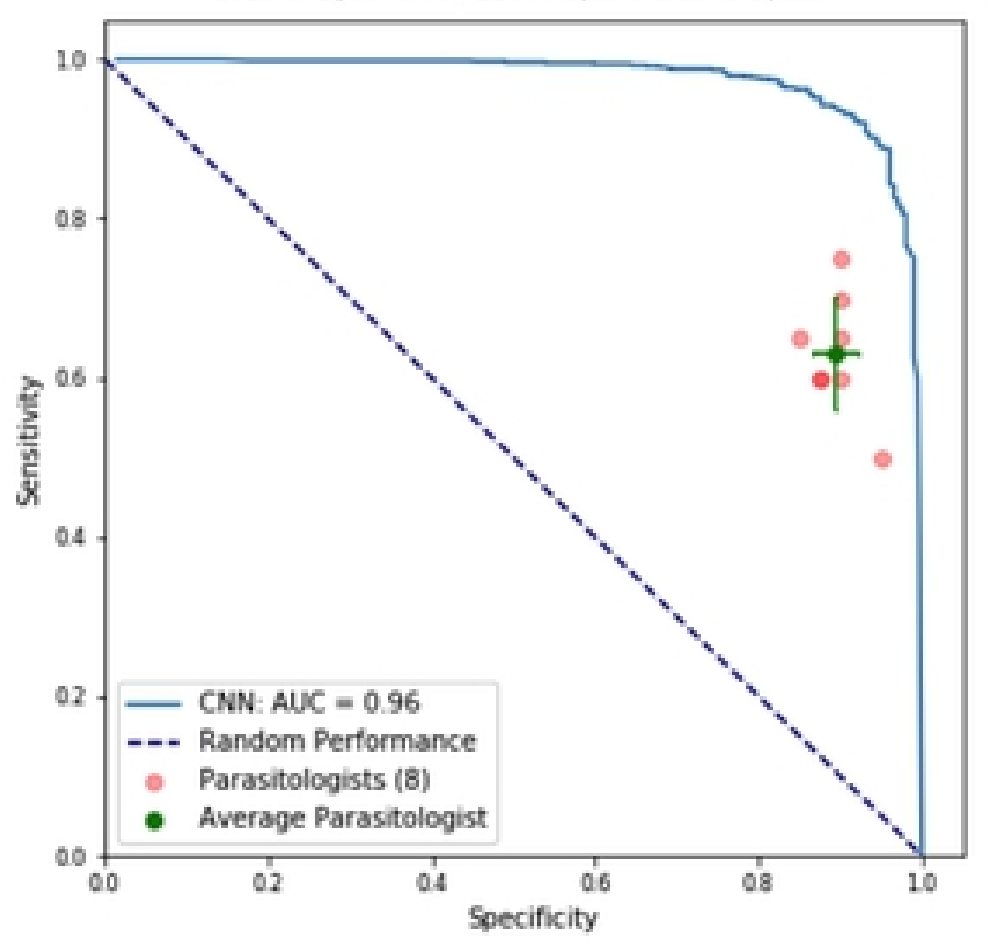

1029 images (CNN); 120 images (Parasitologists)

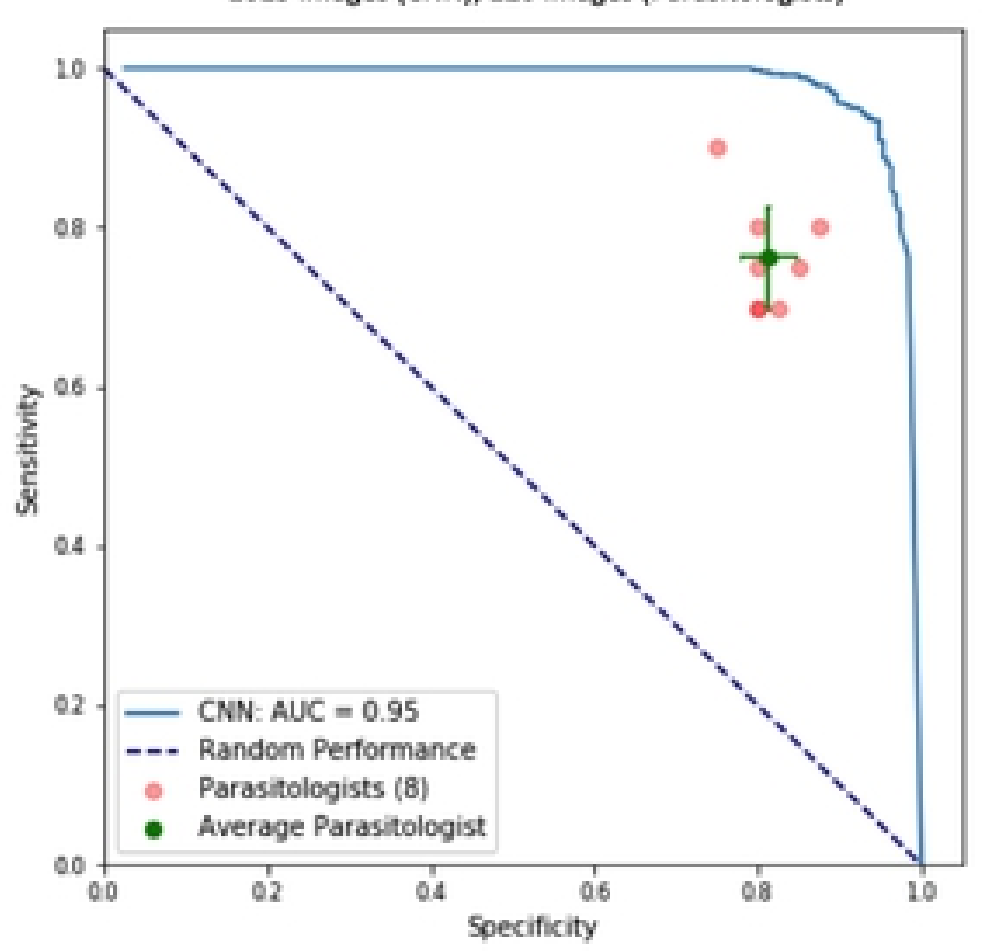

1029 images (CNN); 120 images (Parasitologists)

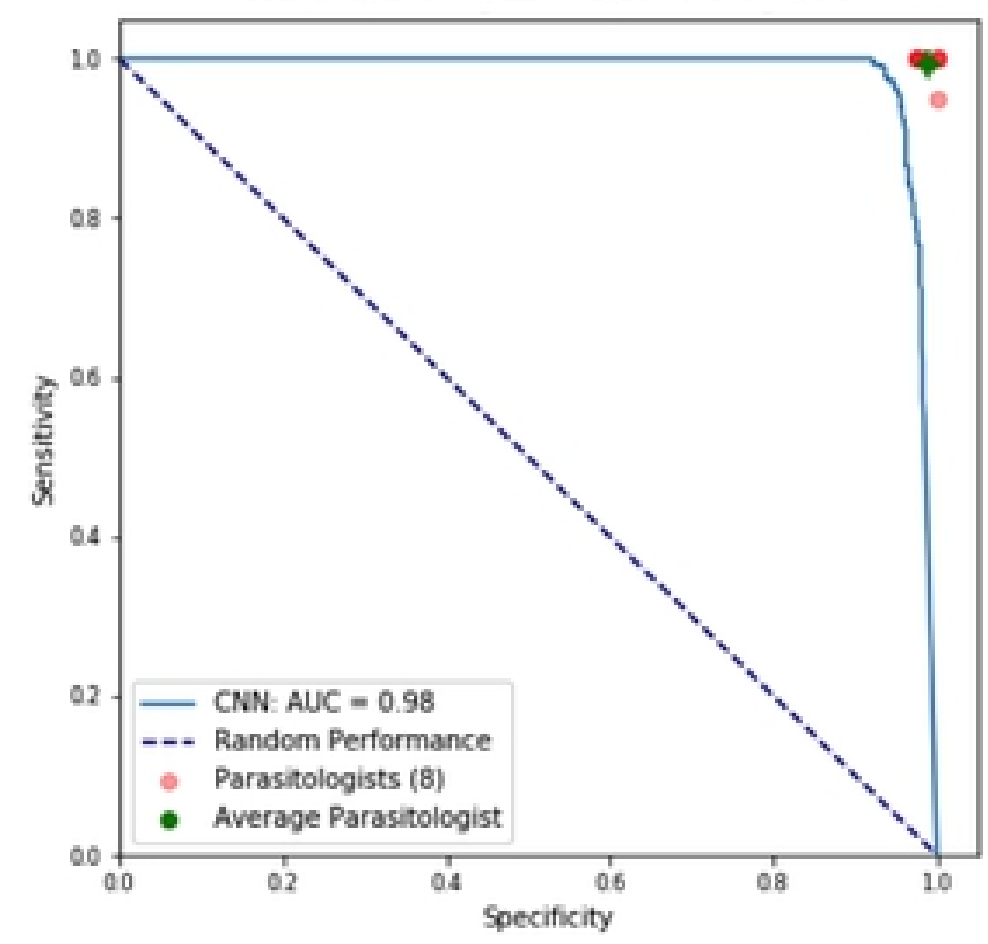

Figure 4 